\title{
A methodology for setting practice criteria in healthcare
}

\author{
D Zaenger \& A F Al-Assaf
}

Dennis Zaenger, MPH, Scientist at the USAID Quality Assurance Project, Bethesda, Maryland, USA

A F Al-Assaf, MD, MPH, MS, CQA, FRSH, Associate Professor and Director of the MPH Degree Program, Department of Health Administration and Policy, University of Oklahoma Health Sciences Centre, Oklahoma City, Oklahoma, USA

Address for correspondence: A F Al-Assaf, MD, College of Public Health, University of Oklahoma Health Sciences Centre, PO Box 26901, Oklahoma City, OK 73190, USA

\section{Key words}

Indicators; criteria; standards; performance measures

\section{Abstract}

Practice criteria are an important part of health care and have taken a new prominence in the trend to address quality-of-care issues. Once an organisation makes a commitment to addressing its quality-ofcare, it must define 'quality' in operational terms. Practice criteria do just that. The organisation ensures consistent, high-quality services through the correct application of practice criteria. This paper outlines a methodology that has been used in at least three countries to date. Early indications are that it is useful for helping an organisation begin its quality improvement 'journey'.

\section{What are practice criteria?}

Practice criteria, defined broadly, are statements of expectations for the inputs, processes, behaviours and outcomes of health systems. Simply put, practice criteria tell us what we expect to happen in our quest for high-quality health services. Practice criteria are important because they are the vehicle by which the organisation translates quality into operational terms and holds everyone in the system (patient, care provider, support personnel, management) accountable for their part. Practice criteria also allow the organisation to measure its level of quality. Practice criteria, indicators, and thresholds are the elements that make a quality assurance system work in a measurable, objective, and qualitative manner (Donabedian, 1980).

\section{Why use practice criteria?}

In every process there is a certain amount of variation. In every task we perform, we vary the way it is done each time. Variation is natural and is to be expected in every process of health care. However, through continuous quality improvement techniques, health workers can increase their knowledge of and control over variation in the health care system (Deming, 1986; Berwick, 1991). Practice criteria help to reduce variation by defining what the organisation expects for the day-to-day inputs, processes, and outcomes of healthcare.

For example, input practice criteria for open-heart surgery help to ensure that surgeons have the necessary and appropriate equipment and staff needed to perform the procedure. Process practice criteria, such as guidelines and protocols, help to ensure that the surgeon is using current, up-to-date techniques and technology. Outcome practice criteria define what the organisation expects as results for the procedure.

Step 1: Identify a function or system

When starting to develop practice criteria, the organisation will need to identify systems or subsystems requiring practice criteria and select one or two that are high priority. These systems are the clinical and non-clinical functions that the organisation engages in regularly. Some primary care examples are acute respiratory infection case management, maternal and child care services, and immunization services. Some hospital-based clinical examples are the performance of caesarian sections and emergency care services. Some non-clinical examples are patient admissions and the use and maintenance of medical records.

Step 2: Identify a team or a panel of experts

These teams should include the right people in order to address issues 
necessary to complete this task (Scholtes, 1988; Brassard, 1989). The 'right' people are those who are best qualified by virtue of their experience, training, and role in the organisation. They are the people who are most involved or most knowledgeable about the function or system. In particular, consider who is involved with each step of the function or system, consider including a technical expert, and consider including someone of authority within the organisation.

Step 3: Identify the inputs, processes, and outcomes

The team or panel of experts must identify the elements for each of the components of the function or system. These are the inputs required to make the processes happen, the processes that are necessary for the expected outcomes to occur, and what is expected as an outcome(s) for the function or system.

\section{Step 4: Define the quality characteristics}

Quality characteristics are the distinguishing attributes of inputs, processes, or outcomes that the organisation or team decides are essential for how it defines quality health care. They are the traits or features by which we judge the quality of health care elements. For example, a team of physicians and laboratory technicians may use 'timeliness' as a characteristic of quality (among others) when setting or evaluating practice criteria for hospital diagnostic tests. Once the team understands and agrees on a quality characteristic, it can then define a standard for it. In this example, the team's next step is to define what it means by 'timeliness' in measurable terms.

\section{Step 5: Develop/adapt practice criteria}

Once the team has decided the quality characteristics for the elements of a function or system, then it must decide which quality characteristics require practice criteria, and then set the practice criteria. A team may decide it does not need a practice criteria for all quality characteristics, and instead focus on what it feels is most important.

Certainly, the team may have to choose a specific format for drafting the practice criteria. Common formats may include statements, algorithms, case management plans, critical paths (Coffey et al, 1992), clinical care protocols, etc. Once the format

FIGURE 1

\section{Six-step process in setting practice criteria}

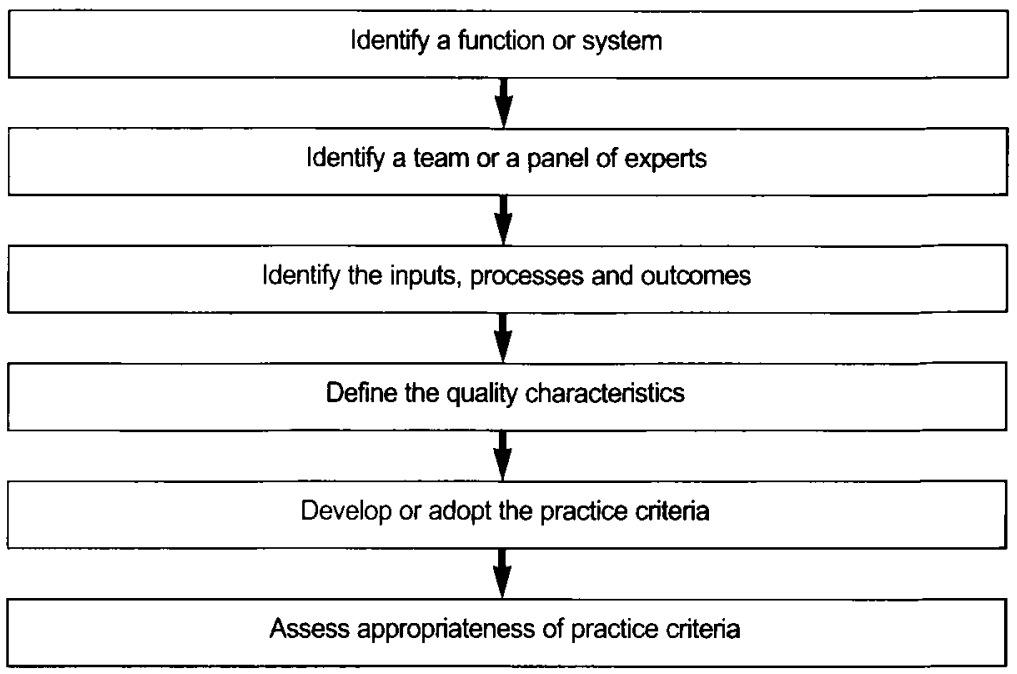

is selected then the team may have to review the literature for similar practice guidelines, or confer with experts, adopt other experiences, or review past experiences.

Step 6: Assess appropriateness of practice criteria

Practice criteria should be assessed to ensure that they are appropriate for the organisation. The team or the organisation should determine if the standards are valid, reliable, clear, and applicable before they are disseminated. All too often, health organisations develop or adopt practice criteria with little or no assessment. Consequently many practice criteria are not appropriate or unrealistic and are simply not followed by intended users. In general, the assessment should be carried out on a small scale, using qualitative rather than quantitative data when necessary.

Additionally, the assessment should determine if the standards have the characteristics described below (IOM 1990). If they do not, then the team should revise the practice criteria and reassess them to ensure that they meet these criteria:

- Assess practice criteria for validity. Assessment should determine if there is a strong demonstrated relationship between the practice criteria and the desired result it represents.

- Assess practice criteria for reliability. Assessment should determine if the same results occur each time the practice criteria are used, i.e., practice criteria measure reproducibility.

- Assess practice criteria for clarity. Assessment should determine if the practice criteria are written in clear, unambiguous terms so that the workers who use the practice criteria do not misinterpret them.

- Assess for applicability. Assessment should determine if the practice criteria are realistic and applicable given the available resources and training of the health care workers responsible for complying with them.

Of course there are numerous challenges to setting practice criteria and one should take into consideration the resistance that may surface from healthcare workers. Some professionals may see these criteria as an impingement on their subjective judgment that they have developed through their practice. There is also the litigious aspect where practitioners may see practice criteria as 'cook book' medicine and not complying 


\section{Setting practice criteria in healthcare}

\section{FIGURE 2}

\section{Quality assurance cycle}

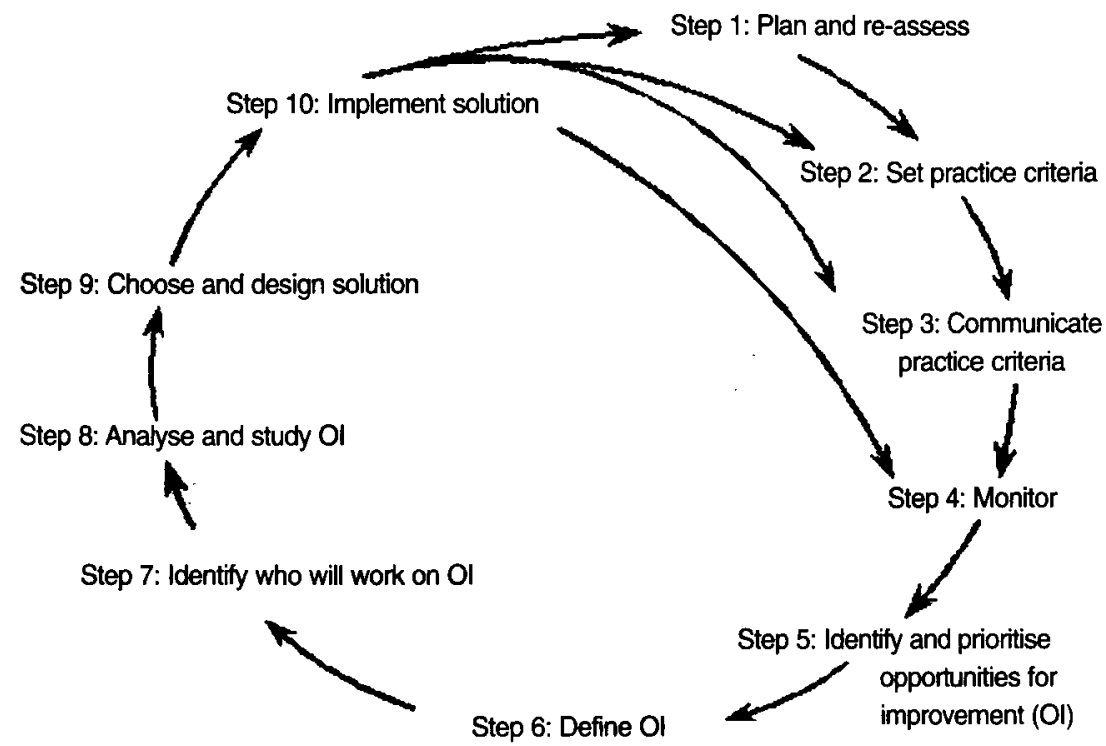

Adapled from the USAID QA Project

with them may bring a law-suit in cases with adverse outcomes (Al-Assaf and Schmele, 1993). Another challenge is identifying appropriate resources to develop these criteria.

\section{Enhancing the Quality of Practice criteria}

The USAID-funded Quality Assurance Project has developed a 10-step approach based on its experience working with health services in developing countries, and based on some US models used in healthcare quality management (Diprete-Brown et al, 1993). (Figure 2) Setting practice criteria is an integral part of this cycle, followed by communicating practice criteria, then monitoring compliance via indicators. Through monitoring, gaps are identified between what is expected to happen in health care, vis-a-vis practice criteria, and what is currently happening. Teams are then assigned to analyse these gaps, identify and implement solutions, and make recommendations to the organisation for adopting the solutions on a wider basis. This often entails modifying, enhancing, or updating practice criteria so that the organisation's expectations for quality are met.

\section{Conclusions}

Setting practice criteria is a necessary component of defining and improving quality of health care. Through practice criteria, an organisation defines what it expects for the inputs, processes, and outcomes of the services it provides. They are an instrumental part of monitoring the quality of care and identifying problems and measuring improvements in health care service delivery. Through periodic updating and modifications, they become a part of an organisation's cycle of continuous quality improvement.

\section{References}

AL-ASSAF A F. and SCHMELE J A (1993). The Textbook of Total Quality in Healthcare. Florida: St. Lucie Press

BERWICK D M (1991). Controlling variation in health care: a consultation from Walter Shewhart. Medical Care, 29(12), 1212-1225

BRASSARD M (1989). The Memory Jogger Plus. GOAI/QPC

COFFEY R J, et al (1992). An introduction to critical pathways. Quality Management in Health Care, 1(1), 45-54

DEMING W E (1986). Out of crisis. Cambridge, Massachusetts: Massachusetts Institute of Technology

DiPRETE-BROWN L, et al (1993). Quality assurance of health care in developing countries. The Quality Assurance Methodology Refinement Series, The Quality Assurance Project

DONABEDIAN A (1980). Explorations in Quality Assessment and Monitoring, Vol I: The Definition of Quality and Approaches to its Assessment. Ann Arbor: Health Administration Press

INSTITUTE OF MEDICINE (1990). Clinical Practice Guidelines: Direction for a New Program. (Eds. Field M J, et al) Washington, DC: National Academy Press

SCHOLTES P R (1988). The Team Handbook: how to use teams to improve quality. Joiner Associates 